\section{Outreach and Partnership Oncology Center Program Model in Eastern Province, Dammam, Saudi Arabia (Out-East)}

\section{Abstract}

The overall goal of King Fahd Specialist Hospital Oncology center outreach program is to bring cancer care delivery to individuals in their own communities, thereby generating a broadly applicable evidence base that contributes to improved patient outcomes and a reduction in cancer disparities through establishment of clearly defined and progressively implemented policy of evidence-based practices, utilizing local resources through a transfer of knowledge and management responsibilities to local healthcare professionals.

\section{Abdulkhalek H*, Alsaqer O, Ibn Shamsah F and Alhashmi $\mathrm{H}$}

\begin{abstract}
Oncology Center, King Fahad Specialist Hospital-Dammam, Eastern province, Saudi Arabia
\end{abstract}

\section{*Corresponding author: Abdulkhalek H \\ झ hosam@consultant.com}

MD, MRCPUK, MSc, MRCPUK Medical Oncology, CPHQ, Quality Program Director of Oncology Center, King Fahad Specialist Hospital-Dammam, Eastern province, Saudi Arabia.

\begin{abstract}
Citation: Abdulkhalek $\mathrm{H}$, Alsaqer $\mathrm{O}$, Ibn Shamsah F, Alhashmi H (2017) Outreach and Partnership Oncology Center Program Model in Eastern Province, Dammam, Saudi Arabia (Out-East). J Hosp Med Manage Vol.3 No.3:23
\end{abstract}

Received: December 15, 2017; Accepted: December 27, 2017; Published: December 30, 2017

\section{Definitions/Abbreviations}

KFSH-D: King Fahad Specialist Hospital-Dammam.

Citrix Systems creates software for use on personal computers and other devices, that allows the individuals of an enterprise to work and collaborate remotely regardless of device or network (i.e., enables KFSH-D staff in outreach hospitals to remotely connect to the KFSH-D network).

Oncology Center Outreach Program: A systematic attempt to provide/deliveroncologyhealthcare services beyond conventional hospital limits to particular segments of a community who might not otherwise have access to these services.

KFSH-D Oncology Outreach Clinic: A KFSH-D outpatient clinic set up at another healthcare facility and staffed by KFSH-D physicians, nurses, pharmacists.

\section{Policy}

The director of the Oncology Center is responsible for initiating the establishing of new oncology outreach program sites [1-4].

\section{Planning}

Assessment and identification of location of outreach services. Ideally, this assessment can be done with minimal new data collection. Efforts should be made to use available data such as the most recent demographic and health data and Ministry of Health $(\mathrm{MOH})$ electronic information system.
Proposal for an oncology outreach program should answer the following questions:

- Are there under-served populations in the program area (national, regional, community-level), and if yes, where are they located?

- What are the main barriers to oncology services for these populations?

- Is there a lack of access to oncology services?

- Is there a lack of trained providers to offer oncology services for these populations?

- Is an oncology outreach program possibly an effective way to address these barriers and to reach these populations?

Identification of Resources (i.e., what resources are available and what resources are needed):

- Availability of trained staff: physicians, nurses, pharmacists and oncology coordinators.

- Training of allocated outreach site clinical staff will be planned and arranged by the Director of Oncology Center and Chairman of the Oncology Outreach Program, in collaboration with the outreach site. 
- Methods of transportation.

- Cost-effectiveness analysis.

Building partnership and planning meetings with the key stakeholders:

- Director of the Oncology Center, KFSH-D.

- Chairman of the Oncology Outreach Program, KFSH-D.

- Chairs of the Adult Medical Oncology Department, the Adult Hemato-Oncology and Stem Cell Transplant (SCT) Department, the Pediatric Hematology-Oncology and Stem Cell Transplant (SCT) Department, and the Palliative Medicine Department, KFSH-D.

- Oncology Outreach Program coordinator, KFSH-D.

- KFSH-D representatives from Pharmaceutical Care Services; Nursing Services, Healthcare Informatics Administration (Information Technology); Laboratory Services; Mail Services, Transportation Services and Finance Services.

- Collaborative and planning meeting with partners and stakeholders from the specific allocated outreach site.

Development of action plan:

- Location of outreach services (must be within the Eastern Province).

- Frequency of outreach services.

- Composition of outreach services delivery team.

- Type and number of training activities (such as how many local staff (physicians, nurses, and pharmacists) needs to be trained by KFSH-D).

- Supply of medications.

- Follow-up and continuity of care activities: A mechanism for the community providers to contact/liaise with the KFSH-D Outreach Program Coordinator when needed.

Implementation includes the following:

Scheduled Services such as locations, dates, and staff for each Oncology Outreach Clinic visit.

Creating a Monitoring Plan for the Oncology Outreach Program:

- Oncology Outreach Program Coordinator will collect program data and submit data monthly to the KFSHD Director of Oncology Center and Chairman of the Oncology Outreach program. Data will be sent quarterly to the Decision Support Department (Quality and Strategic Planning Administration).

- Within 48 hours of returning from a visit, the program team (i.e., visit team leader) will complete a visit report on which is noted the number of patients that were seen in the Oncology Outreach Clinic, demographic data and diagnosis for each patient, and any scheduled future follow-up visits.

Contiual imrovement through reulr progress meetings with specific outreach program site partner to:
- Ensure smooth implementation of the project.

- Discuss and address any problems or challenges.

- Provide refresher training and updates.

\section{Procedures}

1. The Oncology Center Outreach Program will be established and initiated in a specific site allocated for the program after approval from the KFSH-D Director of Oncology Center and Chairman of the Oncology Outreach program.

2. Announcements regarding establishment and initiation of the program will be done by the specific allocated site partner (i.e., any hospital/healthcare facility in the Eastern Province).

The oncology outreach program coordinator will coordinate with the KFSH-D outreach program coordinator to:

- Arrange meetings with KFSHD stakeholders as identified above under policy.

- Arrange collaborative meetings at the specific allocated outreach site clinic.

- With assistance from the Healthcare Informatics Administration, open a new outpatient oncology clinic in Medica-Plus (hospital Health Information System (HIS)), named according to the specific allocated outreach site clinic.

- Selecting patients: types and characteristics of patients will be seen in the Oncology Outreach Clinic.

Primary physician will complete patient selection by:

- Explaining Oncology Outreach Program to the patient.

- Confirming that the patient lives within/near to the specific allocated outreach site clinic.

- Ensuring that the patient's medical record number and medical report are sent (through hospital internal email), along with any other relevant details, to the Oncology Outreach Program Coordinator.

Oncology Outreach Program Coordinator will collect patient demographic data (age, gender, etc., from the patient's KFSH-D medical record), contact numbers, make the necessary outreach clinic appointments, and communicate with the patient through text messages and phone calls.

\section{Timeline for oncology outreach clinic}

Four days before date of the outreach clinic, the Oncology Outreach Program Coordinator will prepare a complete list of selected patients that includes name of the outreach site clinic, date and time of outreach clinic, patient names, medical record number, patient identification numbers, diagnosis, contact number and name of the patient's primary physician. List will be sent via KFSH-D hospital internal email to the following:

- Medical Records Section (Health Information Department).

- KFSH-D Outreach Physicians. 
- KFSH-D Pharmaceutical Care Services.

- Specific allocated outreach site clinic coordinator. Outreach site clinic coordinator will communicate with patients and prepare patient files and clinics.

- Three days before the outreach clinic, KFSH-D Medical Records Section will prepare the selected patient medical records/files for review by the outreach clinic physician.

- Two days before the outreach clinic, the physician will review the patient's medical records and make the necessary medication renewals in Medica-Plus.

- Two days before the outreach clinic, the Oncology Outreach Program Coordinator will contact all selected patients by phone to confirm their appointments, and check that they complete/have completed any ordered lab tests before clinic date.

Oncology outreach program coordinator will:

- Request transportation by completing the vehicle request form, forty-eight (48) hours prior to the oncology outreach clinic visit, obtain approval signature from the Ditrector of Oncology Center and send the request to the KFSH-D Transportation Department for action.

- Complete a business trip form at the beginning and end of the mission, obtain approval from the Oncology Center Director and forward the form to the Financial Affairs Administration.

Forty-eight (48) hours prior to the oncology outreach clinic visit, KFSH-D Pharmaceutical Care Services will dispense the prescribed medication ordered by the oncology outreach program physicians, and label medications with the patients demographic data and prescription details (i.e., dose, duration of use and route of administration).

The Oncology Outreach Program Coordinator will ensure that these medications are delivered to the patient during the outreach clinic visit or delivered to the patient through the official courier service.

All critical laboratory results (of specimens collected during the Oncology Outreach Clinic visit) will be communicated to the KFSHD on-call physicians during business days. On-call physician (of the patient's primary service) will document result in the patient's electronic medical record (physician clinical/progress notes in Medica-Plus) and, if needed, contact the patient by mobile phone to take any necessary medical interaction.

Oncology outreach clinic physician will make use of the remote access (Citrix Systems) during the visit.

Physicians will complete the management plan for each patient in each clinic as a reference for the department; and to assist in the statistics (prepared by Oncology Outreach Program Coordinator) to ensure the continuity of care.

The Oncology Outreach Program Coordinator will rebook patients to the relevant KFSH-D outpatient clinic when requested by the oncology outreach clinic physician.

For continuity of care, the Oncology Outreach Program Coordinator will keep a copy of outreach patient clinic notes in the outreach clinic files, and forward original to the KFSH-D Medical Record section to file in the patient's original KFSH-D medical record.

The allocated outreach hospital (where clinic is conducted) will maintain outreach patient files for KFSH-D patients in a separate closed cabinet. These files will contain the outreach physician clinical notes from every clinic visit for each patient, dated and signed by the author.

Oncology outreach clinic physician will prepare a complete report of the outreach clinic visits and submit report to the Oncology Outreach Program Coordinator for review and approval from the Oncology Outreach Program Director. A copy of this report will be sent to KFSH-D Outreach Program office.

The Collected data will be submitted to the quality and stratigic planning admistration of KFSH-D quarterly to be analysed and published.

\section{Responsibility}

1. Director of Oncology Center, Chairman of Oncology Center Outreach Program and the Oncology Outreach Program Coordinator are responsible for implementing and ensuring compliance with this policy and procedure.

2. Chairman of the Oncology Center Outreach Program is responsible for the review and revision of this policy and procedure.

\section{References}

1 Hovig D, Quinn H (2009) Marie Stopes International Mobile Choice Outreach Programme Overview.

2 https://ncorp.cancer.gov

3 https://www.stjude.org/global/international-outreach.html

4 www.kfsh.med.sa/KFSH_Website/Default.aspx 